\title{
A Quasi-Two-Dimensional Model for High-Power RF LDMOS Transistors
}

\author{
John P. Everett, Michael J. Kearney, Hernan Rueda, Eric M. Johnson, Member, IEEE, Peter H. Aaen, \\ Senior Member, IEEE, John Wood, Fellow, IEEE, and Christopher M. Snowden Fellow, IEEE
}

\begin{abstract}
A new quasi-two-dimensional (Q2D) model for laterally diffused MOS (LDMOS) RF power transistors is described in this paper. We model the intrinsic transistor as a series PHV-NHV network, where the regional boundary is treated as a revere biased $p+/ n$ diode. A single set of one-dimensional energy transport equations are solved across a two-dimensional cross-section in a "current-driven" form and specific device features are modeled without having to solve regional boundary node potentials using numerical iteration procedures within the model itself. This fast, process-oriented, nonlinear physical model is scalable over a wide range of device widths and accurately models DC and microwave characteristics.
\end{abstract}

Index Terms - Field Effect transistor (FET), laterally diffused MOS (LDMOS), quasi-two-dimensional (Q2D), transistor model.

\section{INTRODUCTION}

$\mathrm{T}$ HE LDMOS device structure is widely used in silicon FETs for RF and microwave power amplifiers for communication applications, and is the dominant technology for cellular infrastructure. Indeed, LDMOS technologies are now available achieving $73 \%$ efficiency with $23 \mathrm{~dB}$ gain, breakdown voltages of over $110 \mathrm{~V}$, over $1 \mathrm{Watt} / \mathrm{mm}$ gate periphery and operating frequencies up to $3.8 \mathrm{GHz}$. [1]. Fig. 1 shows an LDMOS die in a microwave power package.

Fig. 2 shows a schematic cross-section of a LDMOS transistor. A consequence of its structural asymmetry is that the charge and field distributions associated with the laterally diffused p-channel (PHV) and n-type drift (NHV) regions have complex drain bias dependencies, which presents technology specific difficulties to the various modeling approaches adopted. Generally, semiconductor devices are modeled using equivalent circuit models extracted from DC and microwave measurements or by using simulation software

Manuscript received ….....................2011. First published

J. P. Everett and M. J. Kearney are with the Advanced Technology Institute at the University of Surrey, UK (e-mail: j.everett@surrey.ac.uk). C. M. Snowden is the Vice Chancellor of the University of Surrey.

H. Rueda, E. M. Johnson, P. H. Aaen and J. Wood are with RF Division, Freescale Semiconductor, Inc., Tempe, USA. This work was supported by Freescale Semiconductor Inc., Tempe Arizona, USA.

Color versions of one or more of the figures in this paper are available online at http://ieeexplore.iee.org.

Digital Object Identifier

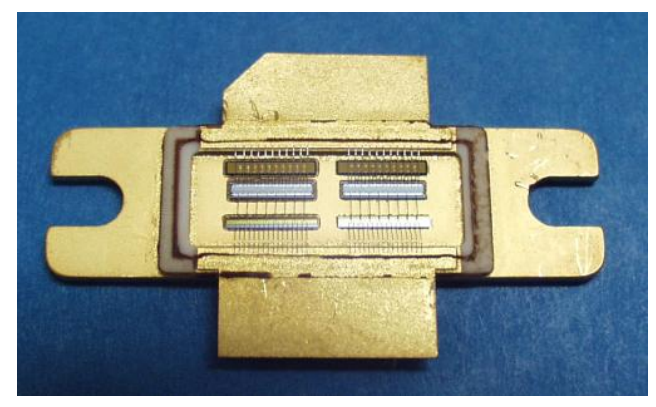

Fig. 1. Photograph of an open LDMOS microwave power transistor showing the complex internal matching networks and the LDMOS die (courtesy of Freescale).

based on physical descriptions of the transistor and the transport parameters of electrons and holes in the semiconductor material. More recently, models have been developed that account for the electro-thermal and electromagnetic interactions of devices that have been shown to be particularly important for larger power transistors [2].

Although many studies have attempted to model different LDMOS architectures using sub-circuit models, here we focus on the development of consistent physical compact models for all specific device characteristics. Full two-dimensional (2D) numerical MOSFET models do allow insight into the device physics but their intensive computational requirements generally render them too cumbersome and slow for most circuit simulation applications [3]. The purpose of physical compact models on the other hand is to describe terminal attributes, including charge, current and capacitances, by a simplified single set of consistent, continuous and accurate physics-based equations, making them faster and more robust for circuit simulation [4]. A quasi-two-dimensional (Q2D) modeling approach, used successfully to model compound semiconductor MESFETs and HEMTs [5]-[8], achieves the simplicity, speed and robustness of physical compact models, while providing an accurate model by taking into account the most important physical phenomena occurring in the device.

The general modeling strategy for the physically-based compact models for LDMOS is to divide the device into two components: an intrinsic MOS channel and a drift region, where the former is modeled as a high-voltage MOS transistor and the latter a non-linear resistor and/or JFET. The drift region has often been subdivided into two or more regions: an 


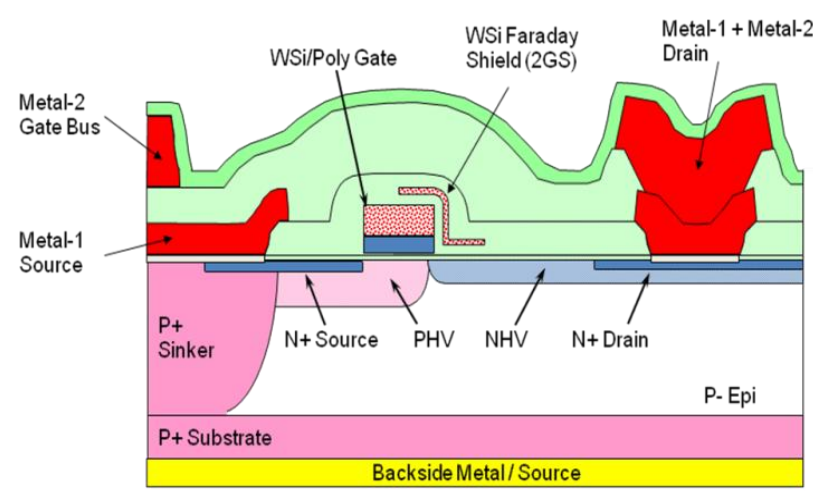

Fig. 2. Schematic cross-section of a LDMOS power transistor.

accumulation region due to gate overlap, a drift region with cylindrical junction, an upper surface accumulation/depletion region under the field plate and a drift region without field plate [9]-[15]. Another key feature of these models is the inclusion of internal drain potentials at regional boundaries that are solved by numerical iteration procedures within the model itself.

The two main approaches to modeling the intrinsic transistor are based on the drift-diffusion approximation (neglecting hot carriers) and are centered on either the inversion-charge (IC) or surface-potential (SP) of the MOSFET channel. Here, the drain current and terminal charges are indirect functions of the terminal voltages through either the surface potential or the inversion charge density. Conventional surface potential models are based on the original charge sheet approximation [16] while for the inversion-charge based models, the inversion charge density is approximated using the UCC model [17]. Examples of IC compact models are the Advanced Compact MOSFET (ACM) [18], BSIM5 [19] and EKV [20]. However, it is the SP modeling approach that has taken lead position in recent years and examples include the PSP model [21], itself is a combination of the MM11 [22] and SP [23] models; the core of these models with charge sheet approximation consists of the inversion charge and channel current equations.

Surface potential based LDMOS models have been reported for DC operation only [24], and for the DC and AC domains [25]. The latter combines the low-voltage MOS region with the high-voltage drift region but does not demonstrate scalability. Although good accuracy for DC operation has been achieved by other models, these have lacked accuracy for the AC domain and scalability, especially with NHV length, temperature and device width [26]-[29]. Chauhan et al. present an IC based scalable general high voltage MOSFET model, applicable for any high voltage MOSFET with extended drift region, which is based on the EKV model and includes physical effects such as quasisaturation, impact ionization and self-heating [10].

The Q2D model for LDMOS described here uses a 1D transport model applied across a 2D cross-section. Separating the full 2D device equations into their $x$ and $y$ components allows us to rewrite them in a simplified hydrodynamic,

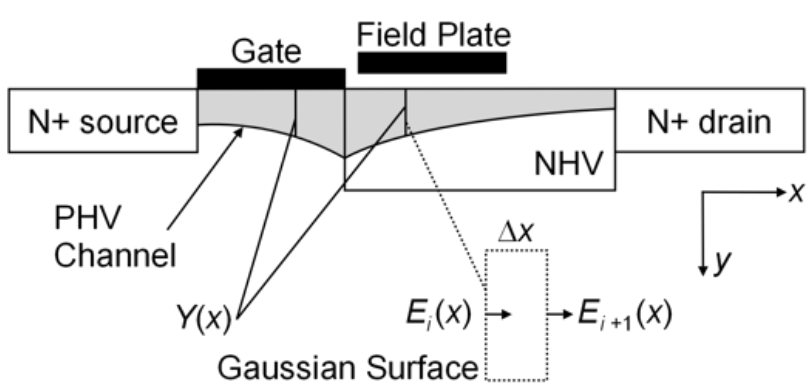

Fig. 3. Domain and structure of Q2D model for the intrinsic LDMOS transistor. The area shaded light grey represents the active channel.

"current-driven" form. The electron energy transport equations are then solved in the intrinsic transistor, which is modeled as a PHV-NHV network where the regional boundary is treated as a revere biased $\mathrm{p}+\mathrm{n}$ diode. The nonlinear algorithm takes account of the PHV and NHV issues discussed previously, does not require numerical iteration of the internal potentials and allows accurate and predictive LDMOS transistor modeling without the need for intermediate measurement and equivalent circuit modeling. Simulation times are fast and this, combined with a low memory footprint, makes the Q2D simulator suitable for device and circuit simulation.

\section{QUASI-TwO-DIMENSIONAL LDMOS MODEL}

\section{A. Basic Assumptions}

Fig. 3 shows a schematic cross-section of the simplified intrinsic LDMOS transistor, which serves as the foundation for the physical Q2D model and simulator described in this section. Doping concentrations in the PHV and NHV regions are approximated from cross-sections towards the surface derived from two-dimensional process simulation [30] of a $7^{\text {th }}$ generation RF LDMOS transistor manufactured by Freescale. We model the intrinsic transistor as a series PHV-NHV network where the former region is treated as a short channel ideal MOSFET and the latter a series combination of the nside space charge and quasi-neutral $(\mathrm{QN})$ regions of a reverse biased $\mathrm{p}+\mathrm{n}$ diode.

Essentially, the Q2D model assumes that the electric field in the active channel region is one-dimensional (1D) while retaining a sufficiently accurate $2 \mathrm{D}$ physical description of the conduction channel. Under these assumptions, and application of Gauss's law to an incremental volume with the uniform cross-sectional area defined by the Gaussian surface and shown in Fig. 3, we can reduce the 2D Poisson equation to 1D slices

$$
\frac{\partial E}{\partial x}=\frac{q}{\varepsilon_{0} \varepsilon_{r}}[N(x)-n(x)]
$$

where $E$ is the electric field, $n(x)$ is the carrier density and $N(x)$ the effective doping density, which is a function of the doping density in the $y$ direction. This is then solved self-consistently with a simplified 1D solution of the hot electron equations in a 
"current-driven" form, where the static drain-to-source current through the device, $I_{D S}$, is assumed constant and the drain-tosource voltage, $V_{D S}$, is given by

$$
\begin{aligned}
& V_{D S}\left(V_{G S}, I_{D S}, T\right)= \\
& \quad \sum_{i}^{L_{P H V}}[E(x) \Delta x]_{i}+\sum_{i}^{L_{N H V}}[E(x) \Delta x]_{i}+V_{Q N} .
\end{aligned}
$$

Here, $V_{G S}$ is the gate-to-source voltage, $T$ the absolute temperature, $L_{P H V}$ and $L_{N H V}$ the length of the PHV and NHV region respectively, $E(x)$ the lateral component of electric field, subscript $i$ represents the step at each incremental length of conduction channel $\Delta x$ and $V_{Q N}$ is the quasi-neutral voltage.

\section{B. The Transport Model}

The transport equation is obtained from the current continuity equation, Gauss's law for an incremental slice and the simplified energy and momentum conservation equations. Simulation boundaries of each slice are defined by the device width $Z$, the total active channel height $Y(x)$ and the slice width $\Delta x$. The intrinsic transistor is divided into slices of width less than the Debye length of the semiconductor material, and the transport model equations for silicon are written in terms of a finite differencing scheme where subscripts $i$ and $i-1$ denote local values at the conduction channel points:

$$
\begin{aligned}
& I_{D S}=q Z Y_{i}(x) n_{i}(x) v_{i}(x)=q Z Y_{i}(x) n_{i}(x) \mu_{i}(x) E_{i}(x), \\
& {\left[E_{i}(x)-E_{i-1}(x)\right] Y_{i}(x)=\frac{q \Delta x}{\varepsilon}\left[N_{i}(x)-n_{i}(x)\right]} \\
& \omega_{i}(x)=\omega_{i-1}(x)+\frac{q \Delta x}{20}\left[21 E_{i-1}(x)-3 \sqrt{A_{i-1}}\right]
\end{aligned}
$$

where

$$
A_{i-1}=40 E_{S S, i-1}^{2}(x)+9 E_{i-1}^{2}(x) .
$$

$\mu(x)$ is the electron mobility, $v(x)$ is the electron velocity, $\omega(x)$ is the average electron energy and $E_{s s}(x)$ is the measured steady state electric field. Here, (3) is the current continuity equation, where the product $Y(x) n(x)$ is the sheet carrier density, (4) is Gauss's law applied to a slice and (5) is the energy conservation equation.

The steady state electric field, at a point $x$, is a function of the average electron energy, doping density and temperature, and is obtained from a curve fit to Monte-Carlo data [31]. The measured field-dependent steady state velocity can be fit by

$$
v_{s s, i}(E)=\mu_{0}^{n} E /\left[1+\left(E / E_{c}\right)^{\beta}\right]^{1 / \beta},
$$

where, from [32], the fitting parameter $\beta=2.57 \times 10^{-2} T^{1.55}$, the critical electric field $E_{c}=1.01 \times T^{1.55}$ and $\mu_{0}^{n}=v_{S a t, S i} / E_{c}$. The saturation drift velocity of electrons in silicon is given by

$$
v_{S a t, S i}=v_{S 0}[1+C \cdot \exp (T / I)]^{-1},
$$

where, from [32], $v_{S 0}=2.4 \times 10^{5} \mathrm{~m} / \mathrm{s}, C=0.8$ and $I=600 \mathrm{~K}$. Rearrangement of the discretized current continuity and Gauss equation produces a quadratic in $E_{i}(x)$, where one of the roots is positive and used to obtain the channel electric field [6]. The hot electron transport equations describing the model are solved in one dimension along the active channel in the PHV and NHV regions. Next, we present details of the active channel height and doping profiles in these regions; note that from here we omit $i$ subscripts for clarity.

\section{The PHV Region}

Under the assumption that in the PHV region the gate area is equal to the active channel area, we obtain a current continuity expression similar to that of (3):

$$
I_{D S}=Z Q_{i n v}(x) \mu(x) E(x),
$$

where $Q_{i n v}(x)$ is the inversion channel charge per unit gate area responsible for current conduction. The charge control element is based on the charge sheet model [33], which assumes that the charges are located at the silicon surface beneath the gate as a sheet of charge with no potential drop or band bending across the inversion layer. The inversion charge density is given by

$$
\begin{aligned}
\left|Q_{i n v}(x)\right| & =\left[V_{G S}-\varphi_{S}(x)\right] C_{o x}-\sqrt{2 \varepsilon_{S i} q N_{A}(x) \varphi_{S}(x)} \\
& =C_{o x}\left[V_{G S}-\left(V_{T}(x)-V(x)\right)\right]
\end{aligned},
$$

where $\varphi_{S}(x)$ is the surface potential, $\varepsilon_{S i}$ is the silicon permittivity, $N_{A}(x)$ is the acceptor impurity density of the ptype (boron-doped) silicon, $V_{T}(x)$ is the threshold voltage, $V(x)$ is the channel potential, $C_{o x}=\varepsilon_{o x} / d_{o x}$ is the oxide capacitance per unit area, and $\varepsilon_{o x} \sim 10^{-11} \mathrm{~F} / \mathrm{m}$ and $d_{o x} \sim 200 \AA$ are the gate oxide permittivity and thickness respectively. A key parameter of a MOSFET device is the threshold voltage, which is defined by

$$
V_{T}(x)=V_{F B}(x)+2 \varphi_{B}(x)+\frac{\sqrt{2 \varepsilon_{S i} q N_{A}(x) \varphi_{S}(x)}}{C_{o x}} .
$$

Here, $V_{F B}(x)$ is the flat band voltage given by

$$
V_{F B}(x)=-\frac{E_{g}}{2}-\varphi_{B}(x),
$$

where the energy gap of silicon is $E_{g} \sim 1.12 \mathrm{eV}$ and the difference between the Fermi and intrinsic levels, $\varphi_{B}(x)$, is

$$
\varphi_{B}(x)=2 \frac{k T}{q} \ln \left(N_{A}(x) / n_{\mathrm{int}}\right)
$$


the intrinsic carrier density $n_{\text {int }}=\sqrt{N_{C} N_{V}} \cdot \exp \left(-E_{g} / 2 k T\right)$. The surface potential is related to the channel potential:

$$
\varphi_{S}(x)=2 \varphi_{B}(x)+V(x) .
$$

Using (13), the conductive channel height at any position $x$ along the conduction channel in the PHV region is readily obtained:

$$
Y(x)=Q_{i n v}(x) / q N_{A}(x)
$$

Consideration of non-uniform doping profiles in the PHV region is important in order to enable the appropriate representation of the fabrication processes used in our model. Although there exists a non-uniform doping profile in the $y$ dimension, to good approximation the profile remains essentially uniform on a scale comparable to the conduction channel height and thus we employ only a laterally decaying surface doping profile for increasing $x$. The net effect of such a profile is a non-uniform conduction channel charge and height.

\section{The NHV Region}

As stated earlier, we model the NHV region as the n-side space charge and quasi-neutral $(\mathrm{QN})$ regions of a reverse biased $\mathrm{p}+\mathrm{n}$ diode where the lateral electric field is the driving force for the reverse saturation drift current. The lateral extension of the depletion layer into the NHV region under negative bias is given by

$$
w_{\text {Lat }}(x)=\varepsilon_{S i} E_{\max }(x) / q N_{D}(x),
$$

where the maximum in the electric field $E_{\max }(x)=E\left(x=L_{P H V}\right)$ occurs at the PHV/NHV boundary.

Assuming no current flows within vertical space charge regions associated with the field plate and reverse biased NHV/P-Epilayer boundary, $w_{F P}(x)$ and $w_{\text {Vert }}(x)$ respectively, the effective cross-sectional area of lateral current flow is $Z Y(x)$ where the active channel height is

$$
Y(x)=d_{N H V}-w_{\text {Vert }}(x)-w_{F P}(x)
$$

for $L_{P H V}<x \leq L_{F P}$. Here, $L_{F P}$ is the length of the field plate and $d_{N H V}$ is the NHV depth, which is assumed constant along its length. The vertical depletion width $w_{\text {Vert }}(x)$ at any point $x$ along the channel [34] is given by

$$
w_{\text {Vert }}(x)=\frac{1}{N_{D}(x)} \sqrt{\frac{2 \varepsilon_{S i} N_{D}(x) N_{A, E P I}\left[V(x)+V_{b i}(x)\right]}{q\left[N_{A, E P I}+N_{D}(x)\right]}} \text {, }
$$

where $N_{A, E P I}$ is the uniform p-Epilayer doping and $V_{b i}(x)$ is the built in potential across the diode:

$$
V_{b i}(x)=\frac{k T}{q} \ln \left[\frac{N_{D}(x) N_{A, E P I}}{n_{\mathrm{int}}^{2}}\right] .
$$

For the case where the surface potential $\varphi_{S}(x) \sim V(x)$ is greater than or equal to the field plate potential $V_{F P} \sim V_{G S}$, we can obtain the vertical depletion approximation due to the field plate from MOS capacitance theory [35]:

$$
w_{F P}(x)=-\frac{\varepsilon_{S i}}{q N_{D}(x) d_{o x}}\left[V_{F P}-\psi_{S}(x)\right]
$$

Note that for simplicity we treat the vertical and lateral depletion processes independently when in reality there exists a complex interaction between them. Also, for the case where $x>L_{F P}$, (17) is applicable in order to calculate $Y(x)$ but with $w_{F P}(x)=0$.

For a quasi-neutral length $L_{Q N}=L_{N H V}-w_{L a t}$, we use a simple expression to calculate the quasi-neutral voltage in the form

$$
V_{Q N}=I_{D S} \rho(x) L_{Q N} / Z Y(x),
$$

where $Y(x)=Y\left(x=w_{\text {Lat }}\right)$. The resistivity of the n-type (arsenicdoped) silicon is approximated from $\rho(x) \sim E(x) / q N_{D}(x) v(x)$ for $x=w_{\text {Lat }}$.

As with the PHV region, consideration of non-uniform doping profiles in the NHV region is important for appropriate representation of the fabrication processes. We incorporate a surface doping profile $N_{D, S}(x)$ that increases to a plateau for increasing $x$. In practice, the NHV doping profile is also non uniform in the $y$-dimension and we accommodate this with the use of an effective lateral profile given by

$$
N_{D}(x)=Y\left(x=L_{P H V}\right) N_{D, S}(x) / d_{N H V} .
$$

\section{E. Physical Simulation Algorithm}

At this point we should stress that along with the Q2D assumptions already mentioned, we also assume that at the PHV-NHV boundary generation and recombination currents are negligible, and Kirchhoff's current and voltage laws hold. The simulation proceeds by solving the discretized model equations over the simulation domain, extending from the edge of the source contact to the edge of the drain contact, for given instantaneous values of source current and gate voltage. To determine the initial electric field at the source end of the PHV channel $E(x=0)$, we use the current continuity equation under the assumptions that the electrons are cool $(v=\mu E)$ in the low-field region. At each mesh point the model variables including the channel charge and conductive channel height are calculated and the steady state electric field, which is a function of average electron energy $w(x)$, is obtained from curve fits to Monte Carlo simulation data. As mentioned above, the channel electric field then is calculated by solving the quadratic form of the discrete version of the coupled current continuity and Gauss equation, and this is numerically integrated to obtain its corresponding channel potential:

$$
V_{i}(x)=V_{i-1}(x)+E_{i-1}(x) \Delta x .
$$

A similar procedure is implemented at each mesh point until the PHV/NHV boundary is reached, thus completing a full simulation of the PHV channel. 
For NHV simulation, the boundary conditions at $x=L_{P H V}$ are determined from the outputs of the PHV simulation. The NHV simulation proceeds across the lateral depletion width $w_{\text {Lat }}$ in a similar form to that outlined for the PHV channel but here the conductive channel height is obtained by different means as previously shown. Finally, the quasi-neutral voltage is obtained from (21) to complete the full simulation of the NHV region. Thus, as shown in (2), the Q2D algorithm gives access to the DC characteristics in a "current-driven" form, where $V_{D S}$ is the channel potential at $x=L_{P H V}+L_{N H V}$ for given instantaneous values of $I_{D S}$ and $V_{G S}$.

Small-signal microwave $S$-parameters can be obtained using a previously reported two-stage multi-frequency extraction scheme [6]. This scheme uses $V_{\mathrm{GS}}$ and $I_{\mathrm{S}}$ as independent variables and the gate current and $I_{\mathrm{DS}}$ as dependent variables, and utilizes short and open-circuit terminations. Extrinsic parasitic impedances are added to the frequency dependent admittance parameters of the intrinsic device to produce corresponding $S$-parameters. The Q2D timedomain LDMOS simulation can also be applied to large-signal simulations by embedding the simulator in a dedicated time domain circuit or commercially available circuit simulator.

\section{DEVICE CHARACTERISTICS}

Validation of the accuracy of the Q2D model for LDMOS has been carried out by comparing the simulation and measurement of current-voltage data from $2.3 \mathrm{~mm}, 4.8 \mathrm{~mm}$ and $9.6 \mathrm{~mm}$ total gate width $(4,8$ and 16 drain fingers $\mathrm{x} 600$ $\mu \mathrm{m}$ unit width respectively) $7^{\text {th }}$-generation HV7 LDMOS transistors. Fig. 4 shows the device's PHV and NHV structure and doping profile; the p-Epilayer doping concentration used was $N_{A, E P I} \sim 10^{15} \mathrm{~cm}^{-1}$. The simulated data were obtained using 188 conduction channel slices and measurements were made on-wafer with the transistors being contacted by groundsignal-ground RF probes. The temperature-controlled wafer chuck maintained the ambient reference temperature at a constant at $25 \mathrm{C}$ throughout the measurements. The measured

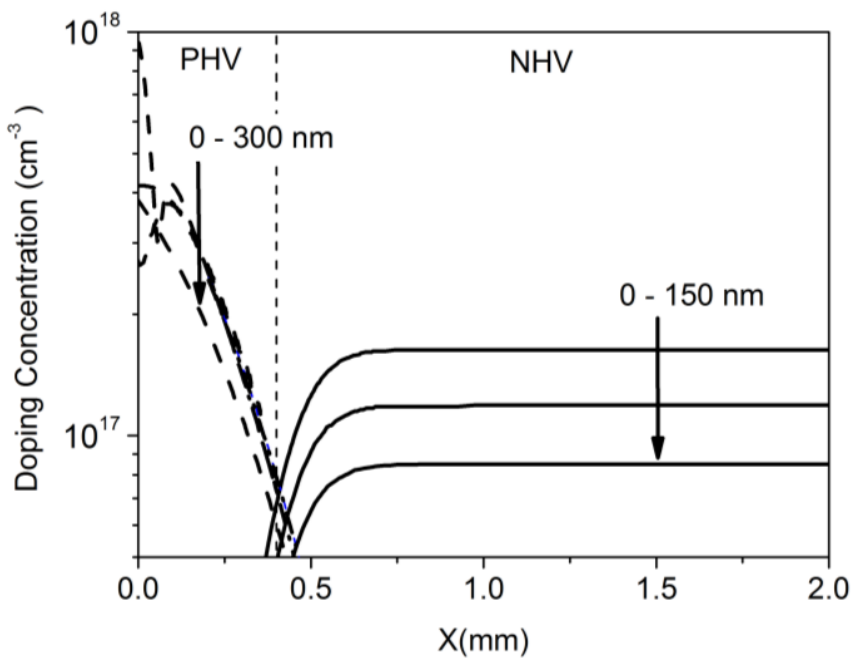

Fig. 4. PHV and NHV structure and doping profile of a $4.8 \mathrm{~mm}$ intrinsic LDMOS transistor for vertical depths $\mathrm{y}=0$ to $300 \mathrm{~nm}$ and $\mathrm{y}=0$ to $150 \mathrm{~nm}$ respectively. The dashed line is the PHV-NHV regional boundary. data were obtained under pulsed conditions to preserve isothermal conditions: the pulse width was $2 \mu$ s with a $400 \mu \mathrm{s}$ pulse period, resulting in a duty cycle of $0.5 \%$. A wide range of gate and drain bias conditions was employed to sample the transistor output characteristics comprehensively.

The raw measured data was referenced to the intrinsic reference plane of the transistor, by de-embedding the extrinsic resistances from the raw measured data at the probe tip measurement plane using

$$
\begin{gathered}
V_{G S, R E F}=V_{G S, R A W}-I_{D S} R_{S}, \\
V_{D S, R E F}=V_{D S, R A W}-I_{D S}\left(R_{D}+R_{S}\right),
\end{gathered}
$$

where $R_{D}$ and $R_{S}$ are the extrinsic drain and source resistances respectively. These extrinsic resistance values were obtained from broadband $S$-parameter measurements of the transistor biased in 'Cold FET' conditions, as outlined in [36]. The measured and simulated data are then scaled to the periphery.

Fig. 5 shows the excellent agreement obtained between the measured and simulated data, over a wide range of gate biases typically found in operation of the transistor. The simulated data have been produced entirely from the Q2D model using physical data of the LDMOS transistor obtained from fabrication process data and from the process simulation, and the transport parameters outlined in Section II.

In Fig. 6, we compare the de-embedded pulsed measured DC $I_{D S}-V_{D S}$ characteristics of the $4.8 \mathrm{~mm}$ transistor with the Q2D simulation data at gate biases close to the threshold voltage, $\sim 2.7 \mathrm{~V}$, and again the agreement between the measured and predicted data is excellent. Fig. 7 compares the measured and Q2D modeled DC transconductance, $G_{m}=\partial I_{D S} / \partial V_{G S}$, for the same transistor at various gate biases which demonstrates further that our Q2D model for LDMOS provides an accurate description of the intrinsic LDMOS DC characteristics over a wide range of bias conditions. Results from a two-dimensional simulation of the $4.8 \mathrm{~mm}$ LDMOS device using the Synopsys Sentaurus Device simulator [30]

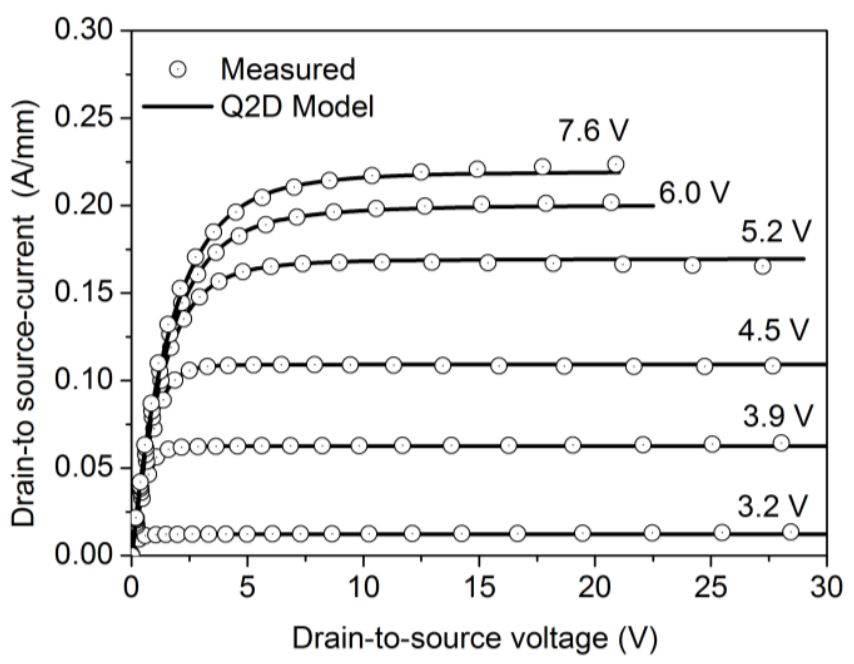

Fig. 5. Comparison between measured and modeled DC characteristics of a $4.8 \mathrm{~mm}$ intrinsic LDMOS transistor. 


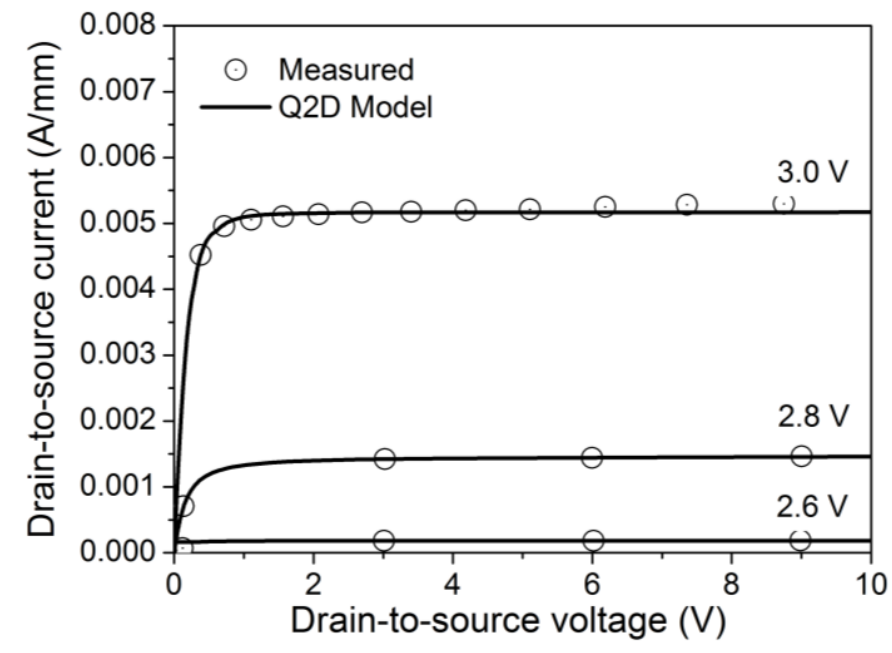

Fig. 6. Comparison between measured and modeled DC characteristic at gate voltages near the threshold voltage.

are superimposed on the lateral electric field Q2D simulation results shown in Fig. 8. Reasonable agreement between the simulations is achieved for parameters that are essentially assumed to be one-dimensional in the active conduction channel.

In Fig. 9 we present the isothermal Q2D model DC characteristics previously shown in Fig. 4, and compare these with thermally degraded curves obtained with the temperature dependent model parameters outlined above. The latter show the negative differential decrease in the drain-to-source current at higher drain-to-source voltages resulting from self-heating effects observed for LDMOS RF power transistors [1], [37].

Model scalability is another important and desirable characteristic of device models. A comparison between measured and simulated DC $I_{D S} V_{D S}$ characteristics of $2.4 \mathrm{~mm}$, $4.8 \mathrm{~mm}$ and $9.6 \mathrm{~mm}$ LDMOS transistors in Fig. 10 demonstrates that the Q2D model scales excellently over a wide range of device widths.

We also report that good agreement between simulated and measured $S$-parameters data is obtained where, for example, at $1 \mathrm{GHz}$ and $2.8 \mathrm{~V}$ gate bias the gain magnitude $\left|S_{21}\right| \sim 10$ and the phase is $\sim 100$ degrees. At this gate bias and $30 \mathrm{~V}$ drain voltage, Q2D large-signal modeling typically produces conservative gate and drain charges of $1.5 \mathrm{pC}$ and $2.5 \mathrm{pC}$ respectively, which is in good agreement with charges obtained through a large-signal Root model extraction from intrinsic measured data [1].

We now highlight the Q2D simulator's low memory footprint and extremely fast computation time, able to evaluate a DC $I_{D S}-V_{D S}$ point in less than $1 \mathrm{~ms}$ on a core i7 PC and thus simulation of 20 cycles of $\mathrm{RF}$ at $1 \mathrm{GHz}$ in less than one second. This is comparable with other nonlinear equivalent circuit models and 10000 s of times faster than commercially available full two-dimensional device simulators such as Synopsys Sentaurus Device [30].

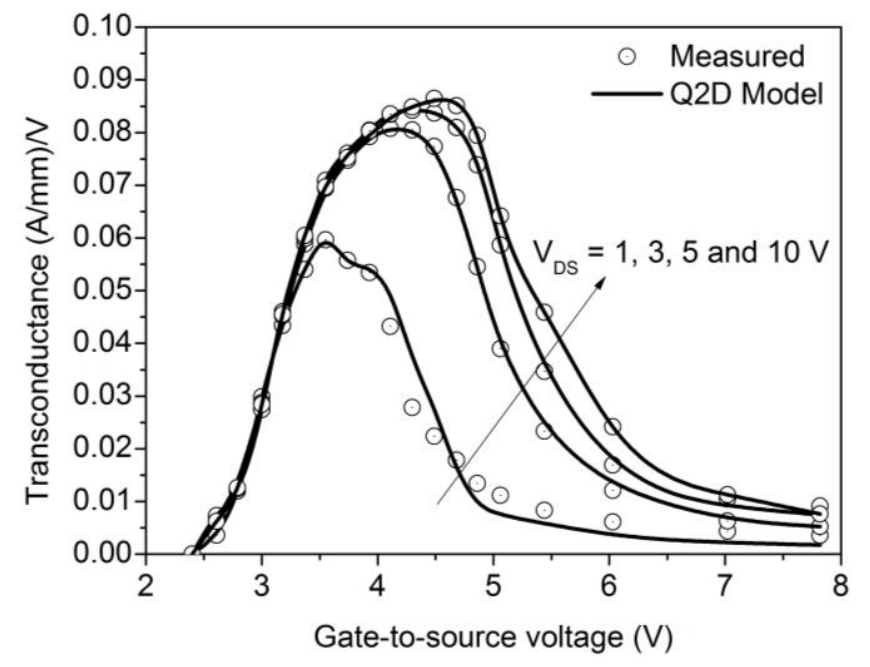

Fig. 7. Comparison between measured and modeled DC transconductance of a $4.8 \mathrm{~mm}$ intrinsic LDMOS transistor.

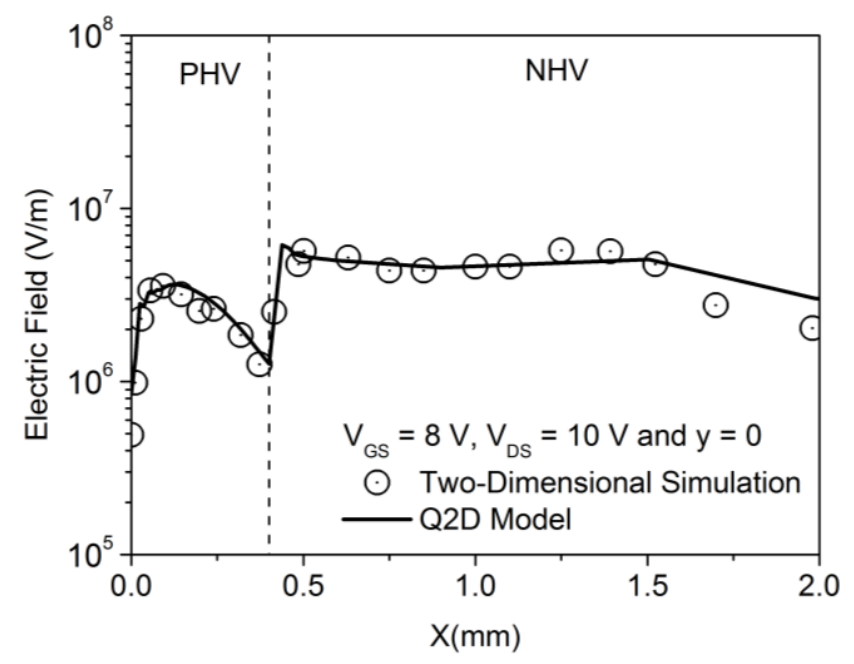

Fig. 8. Q2D lateral electric field simulation results for a $4.8 \mathrm{~mm}$ LDMOS intrinsic transistor. Two-dimensional Synopsys Sentaurus Device simulation results of the intrinsic transistor are superimposed for comparison. The dashed line is the PHV-NHV regional boundary.

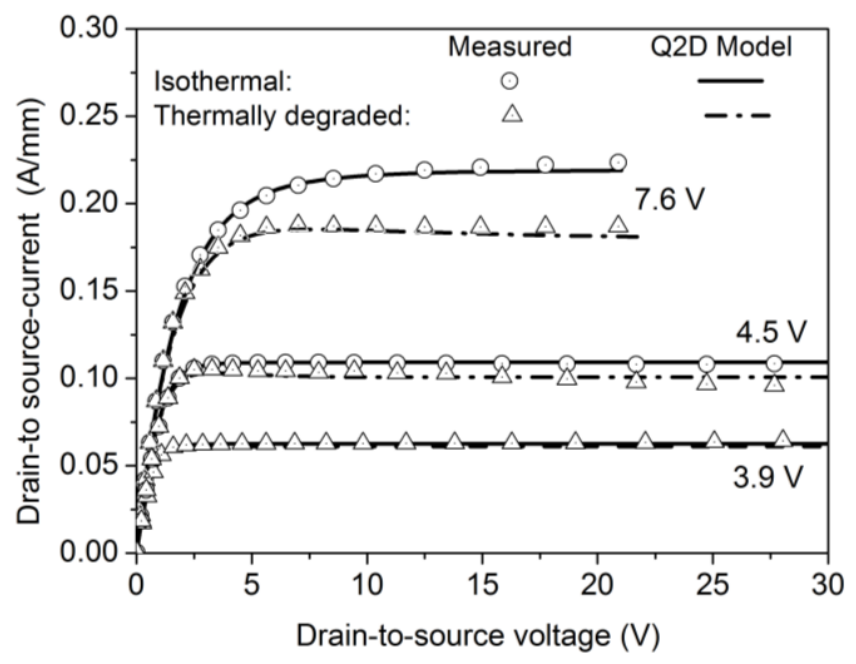

Fig. 9. Comparison between the Q2D modeled isothermal and thermally degraded DC characteristics. 

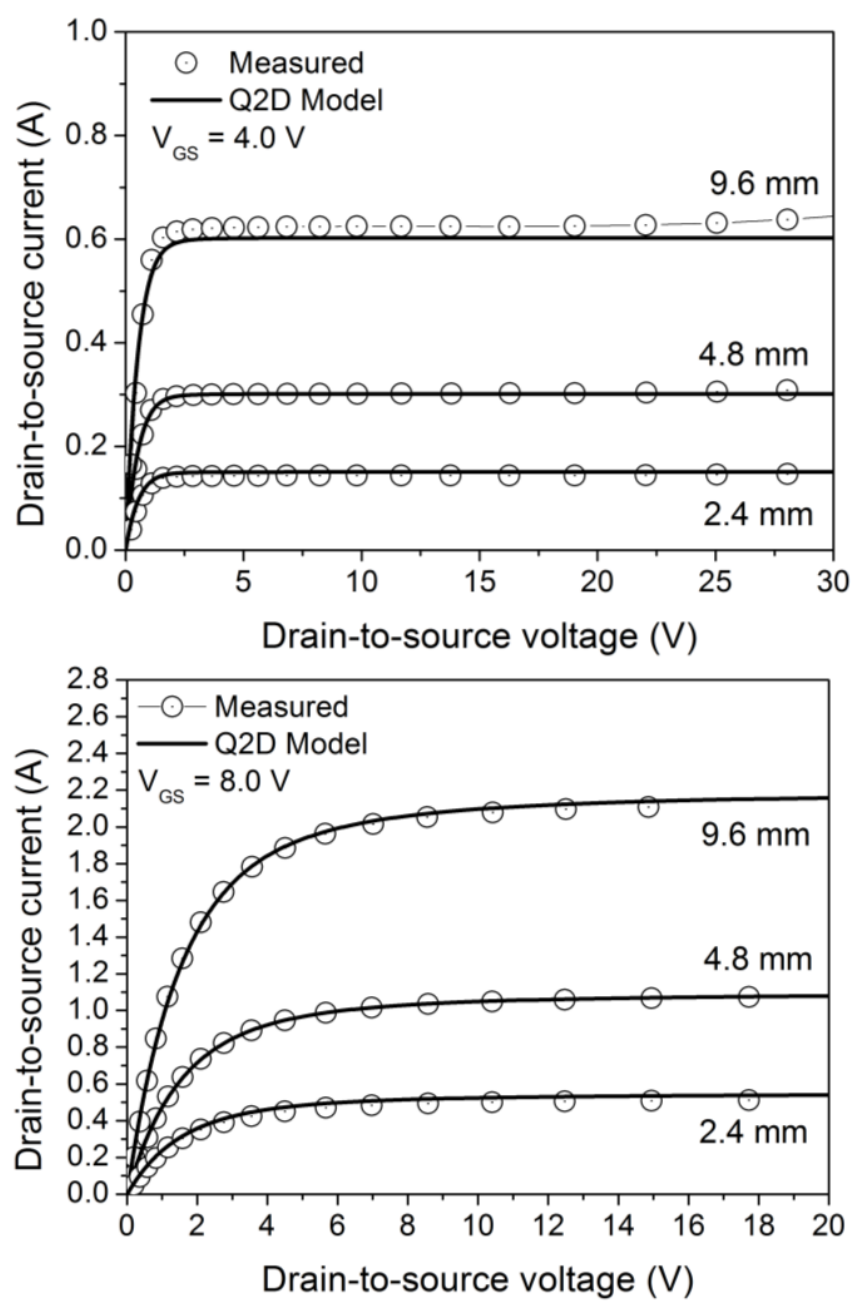

Fig. 10. Comparison between measured and modeled DC characteristics of $2.4 \mathrm{~mm}, 4.8 \mathrm{~mm}$ and $9.6 \mathrm{~mm}$ intrinsic LDMOS transistors at gate biases of $4.0 \mathrm{~V}$ (top) and $8.0 \mathrm{~V}$ (bottom).

\section{CONCLUSION}

We have described a new quasi-two-dimensional physical model for RF power LDMOS FETs, where the intrinsic transistor is modeled as a PHV-NHV network and the regional boundary is treated as a reverse biased $\mathrm{p}+\mathrm{n}$ diode. This "current-driven" model solves simplified 1D hydrodynamic electron energy equations in the active channel, described by its $2 \mathrm{D}$ cross section, and does not require numerical iteration of the internal potentials within the model itself. The Q2D simulator is capable of accurately modeling LDMOS specific features and demonstrates good model accuracy in DC and microwave simulations over a wide range of bias conditions. Moreover, it is scalable over a wide range of device widths and facilitates accurate LDMOS transistor modeling at an execution speed sufficiently fast for circuit simulation applications, which makes it an attractive proposition for predictive modeling.

\section{ACKNOWLEDGMENT}

This work is supported by a direct grant from RF Division, Freescale Semiconductor, Inc.

\section{REFERENCES}

[1] P. H. Aaen, J. A. Plá, and J. Wood, Modeling and Characterization of $R F$ and Microwave Power FETs. Cambridge, UK: Cambridge Univ. Press, 2007.

[2] D. Denis, C. M. Snowden and I. C. Hunter, "Coupled electrothermal, electromagnetic, and physical modeling of microwave power FETs," IEEE Trans. Microw. Theory Tech., vol. 54, no.6, pp. 2465-2470, 2006.

[3] N. Arora, MOSFET Models for VLSI Circuit Simulation Theory and Practice, New York, USA: Springer-Verlag, 1993.

[4] C. Galup-Montoro and M. C. Schneider, MOSFET Modeling for Circuit Analysis and Design. Singapore: World Scientific Publishing Co. Pte. Ltd., 2007.

[5] C. M. Snowden, "Semiconductor device modelling," Rep. Prog. Phys. vol. 48, pp. 223-275, 1985.

[6] C. M. Snowden and R. R. Pantoja, "Quasi-two-dimensional MESFET simulations for CAD," IEEE Trans. Electron Devices, vol. 36, no. 9, pp. 1564-1574, 1989.

[7] C. M. Snowden and R. E. Miles, Compound Semiconductor Device Modeling. London, UK: Springer-Verlag, 1993.

[8] R. Drury and C. M. Snowden, "A quasi-two-dimensional HEMT model for microwave CAD applications," IEEE Trans. Electron Devices. vol. 42, no. 6, pp. 1026-1032, 1995.

[9] M. Yokomichi, N. Sadachika, M. Miyake, T. Kajiwara, H. J. Mattausch, and M. Miura-Mattausch, "Laterally diffused metal oxide semiconductor model for device and circuit optimization," Jpn. J. Appl. Phys., vol. 47, no. 4, pp. 2560-2563, 2008.

[10] Y. S. Chauhan, C. Anghel, F. Krummernacher, C. Maier, R. Gillon, B. Bakeroot, B. Desoete, S. Frere, A. B. Desormeaux, A. Sharma, M. Declercq and A. M. Ionescu. "Scalable general high voltage MOSFET model including quasi-saturation and self-heating effects," Solid-State Electronics, vol. 50, pp. 1801-1813, 2006.

[11] J. Victory, C. C. McAndrew, R. Thoma, K. Joardar, M. Kniffin, S. Merchant, and D. Moncoqut, "A Physically Based Compact Model for LDMOS Transistors,” SISPAD, pp. 270-274, 1998.

[12] K. Lee, J. Yoon, J. Yim, J. Kang, D. Baek, S. Lee, I. Shon and B. Kim, "An improved silicon RF LDMOSFET model with a new extraction method for nonlinear drift resistance," IEEE RFIC Symposium, pp. 153$156,2005$.

[13] Jian-Meng, Shan-Gao, Jun-Ning Chen and Dao-Ming Ke, "The analysis and modeling of on-resistance in high voltage LDMOS," in Proc. 8th Int. Conf. on Solid-State and Integrated Circuit Technology. pp. 13271329,2006

[14] I. Cortés, J. Roig, D. Flores, J. Urresti, S.Hidalgo and J. Rebollo, "A numerical study of field plate configurations in RF SOI LDMOS transistors," Solid-State Electronics, vol. 50 pp. 155-163, 2006.

[15] Mueng-Ryul Lee and Oh Kyong Dwon, "DC modeling of lateral highvoltage MOSFETs for high-voltage integrated circuits," J. Korean Phys. Soc., vol. 40, no. 4, pp. 663-667, 2002.

[16] H. K. Hermann and K. Singhal, "Inversion charge modeling," IEEE Trans. Electron. Devices, vol. 48, no. 8, pp. 1585-1593, 2001.

[17] Y. H. Byun, K. Lee and M. Shur, "Unified charge control model and subthreshold current in heterostructure field effect transistors," IEEE Electron Device Lett., vol. 11, no. 1, pp. 50-53, 1990.

[18] G. Montoro, C. Schneider, M. C. Cunha, A. I. A. de Sousa, F. R. Klimach, H. Siebel, O. Siebel, "The Advanced Compact MOSFET (ACM) Model for circuit analysis and design," (Invited paper) IEEE Custom Integrated Circuits Conf., San José: USA, Set 2007.

[19] J. He, X. Xi, M. Chan, A. Nickenjad and C. Hu, " An advanced surface potential-plus MOSFET model," Proc. Workshop on Compact Modeling, Nanotech, pp. 262-265, 2003.

[20] C. C. Enz, F. Krummenacher and E. A. Vittoz, "An analytical MOS transistor model valid in all regions of operation and low-voltage an low current applications," J. Analog Integr. Circuits Process., vol. 8, pp. 83114, 1995.

[21] G. Gildenblat, X. Li, H. Wang, W. Wu, R. van Langevelde, A. J. Scholten, G. D. J. Smit and D. B. M. Klaassen, "Introduction to PSP MOSFET Model," Nanotech, pp. 19-24, 2005. 
[22] R. van Langevelde, A. J. Scholten and D. B. M. Klaassen, "Physical Background of MOS Model 11,Unclassified report 2003/00239. Available: http://www.semiconductor.philips.com/Philips_Models/.

[23] G. Gildenblat, H. Wang, T. Chen, X. Gu and X. Cai, "SP: An advanced surface-potential based compact MOSFET model," IEEE J. Solid State Circuits, vol. 39, no. 9, pp. 1394-1406, 2004.

[24] A. Aarts and W. Kloosterman, "Compact modeling of high-voltage LDMOS devices including quasi-saturation," vol. 53, no. 4, 2006.

[25] A. Aarts, N. D'Halleweyn and R. van Langevelde, "A surface-potential based high-voltage compact LDMOS transistor model," IEEE Trans. Electron Devices, vol. 52, no. 5, 2005.

[26] C. Anghel, "High voltage devices for standard MOS technologies: characterisation and modeling," Ph.D. dissertation, Lausanne EPFL, 2004.

[27] N. Hefyene, "Electrical characterisation and modeling of lateral DMOS transistor: investigation in capacitances and hot-carrier impact, " Ph.D. dissertation, Lausanne EPFL, 2005.

[28] J. Victory, J. Sanchez, T. DeMassa and B. Welfert, "A static, physical VDMOS model based on the charge-sheet model," IEEE Trans. Electron Devices, vol. 43, no. 1, 1996.

[29] C-Y Tsai, D. E. Burk and K. D. T. Ngo, "Physical modeling of the power VDMOST for computer-aided design of integrated circuit," IEEE Trans. Electron Devices, vol. 44, no. 3, 1997.

[30] Sentaurus TCAD Software, Release D-2010-03, Synopsys, Mountain View, CA, USA.

[31] M. Lundstrom, Fundamentals of Carrier Transport, Second Edition. Cambridge, UK: Cambridge University Press, 2000.

[32] S. M. Sze, Physics of Semiconductor Devices, Third Edition. New Jersey, USA :John Wiley \& Sons Inc. , 2007.

[33] J. R. Brews, "A charge-sheet model of the MOSFET,"Solid State Electronics, vol. 21, pp. 345-355, 1978.

[34] U. Apel, H. G. Graf, C. Harendt, B. Hofflinger and T. Ifstrom, "A 100-V lateral DMOS transistor with a 0.3 -micrometer channel in a 1micrometer silicon-film-on-insulator-on-silicon, IEEE Trans. Electron. Devices., vol. 38, no. 7, pp. 1655-1659, 1991.

[35] Y. Taur and T. H. Ning, Fundamentals of Modern VLSI devices, Second Edition. Cambridge, UK: Cambridge University Press, 2009.

[36] J. Wood, D. Lamey, M. Guyonnet, D. Chan, D. Bridges, N. Monsauret, and P. H. Aaen, "An extrinsic component parameter extraction method for high power RF LDMOS transistors," Microwave Symposium Digest, pp. 607-610, 15-20 June 2008.

[37] C. Anghel, R. Gillon and A. M. Ionescu, "Self-heating characterization and Extraction method for thermal resistance and capacitance in HV MOSFETs," IEEE Trans. Electron Devices, vol. 25, no. 3, pp. 141-143, 2004.

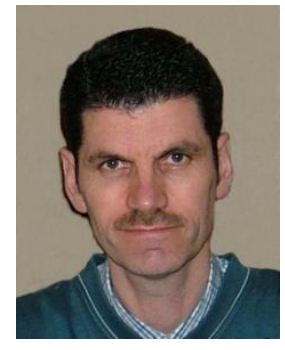

John P. Everett received the B.Sc.(Hons) degree in physics and astrophysics from Queen Mary and Westfield College, London, U.K., in 1994, and in 1998, received the Ph.D. degree and Diploma of the Imperial College from the Imperial College of Science, Technology and Medicine, London, U.K., in high temperature superconductivity and experimental solid state physics respectively.

After graduating, he continued his work at Imperial College as a Postdoctoral Researcher investigating $\mathrm{DC}$ and $\mathrm{AC}$ losses in high temperature superconducting tapes. In 2006, he joined the University of Surrey's Advanced Technology Institute (ATI), U.K., to work on modeling molecular systems with the support of the Daphne Jackson Trust. Since 2008, he has been at the ATI working on the development of compact models for RF power LDMOS devices.

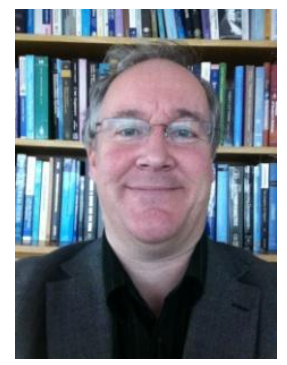

Michael J. Kearney MA PhD FIET FInstP FIMA, is Dean of the Faculty of Engineering and Physical Sciences at the University of Surrey, UK. Born in 1962, he attended St. John's College Oxford in 1982 to study physics. A PhD in theoretical solid state physics followed at the University of Warwick in 1988, whereupon he joined the Long Range Research Laboratory at the GEC Hirst Research Centre, London. After serving a period as Manager of that Laboratory, he left in 1995 to join the Department of Electronic and Electrical Engineering at Loughborough University, serving as Head of Department between 1997 and 2000. He joined Surrey in 2002 as the inaugural Director of the Advanced Technology Institute, and was appointed Head of the School of Electronics and Physical Sciences in 2005. In 2007 he assumed the role of Dean. He has over 100 publications many of which are devoted to modelling III-V devices for RF and microwave applications (e.g. mixers, detectors, sources), SiGe FETs (particularly p-type devices), and thin-film solar cells.

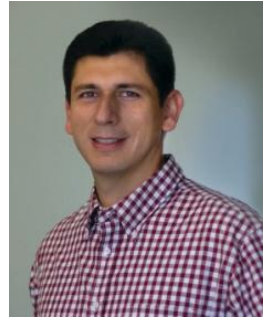

Hernan Rueda received the B.S., M.S., and Ph.D. degrees in electrical engineering from the University of Florida, Gainesville, FL in 1992, 1994, and 1999, respectively. His Ph.D. thesis involved the modeling of mechanical stress in silicon isolation technology and its influence on device characteristics.

In 1999, he joined Motorola Semiconductor where he began working in process and device simulation of SiGe HBT BiCMOS technology. He is currently involved in RF-LDMOS device technology development at Freescale Semiconductor in Tempe, Arizona, USA.

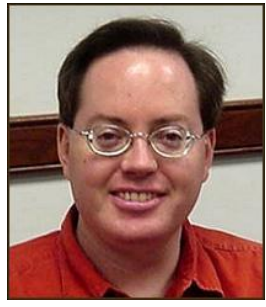

Eric M. Johnson (M'92) received the B.S. degree in electrical engineering from the University of Michigan, Ann Arbor, MI, in 1989, and the M.S. degree in electrical engineering from the National Technological University, Fort Collins, $\mathrm{CO}$ in 1994.

In 1989 he joined Motorola (now Freescale Semiconductor), working primarily in the area of $\mathrm{RF}$ and microwave device modeling and characterization for III-V devices. Since 2007, he has been a member of the RF Division, Freescale Semiconductor Inc., Tempe, $\mathrm{AZ}$, where he has been responsible for RF LDMOS device modeling and characterization. His areas of interest include measurement techniques, measurement automation, and nonlinear device modeling. He has authored or co-authored a number of papers in the areas of RF and microwave modeling and measurements.

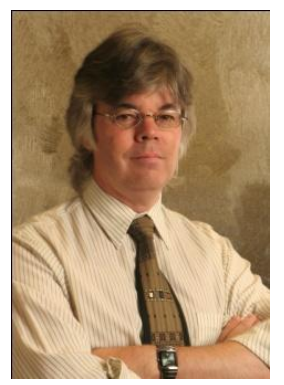

John Wood (M'87, SM'03, F'07) received B. Sc. and $\mathrm{Ph}$. D. degrees in Electrical and Electronic Engineering from the University of Leeds, in 1976 and 1980, respectively. He is a Senior Member of the Technical Staff in the RF Division of Freescale Semiconductor. His areas of expertise include the development of nonlinear compact device models and behavioral models for RF power transistors and ICs, the understanding of the impact, characterization, \& control of nonlinearities in high-efficiency PAs. From 1997--2005 he worked in the Microwave Technology Center of Agilent Technologies, developing large-signal and biasdependent linear FET models for mm-wave applications, and nonlinear behavioral models using LSNA measurements and nonlinear system identification techniques.

$\mathrm{He}$ is author or co-author of over 100 papers and articles. He is a Fellow of the IEEE, and a member of the Microwave Theory and Techniques, and Electron Devices Societies.

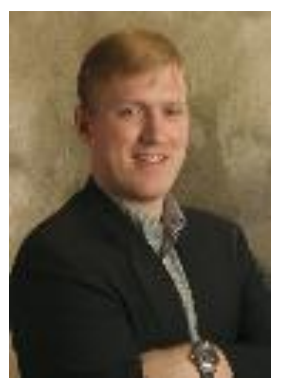

Peter H. Aaen (S'93, M'97 SM'09) received the B.A.Sc. degree in Engineering Science and the M.A.Sc. degree in Electrical Engineering, both from the University of Toronto, Toronto, ON., Canada, and the Ph.D. degree in Electrical Engineering from Arizona State University, Tempe, AZ., USA, in 1995, 1997, and 2005, respectively. $\mathrm{He}$ is the Manager of the RF Modeling team of the RF Division of Freescale Semiconductor, Inc, Tempe, AZ, USA. His areas of interest include the development and validation of microwave transistor models, passive component modeling, and the electromagnetic simulation of complex packaged environments. He has co-authored Modeling and Characterization of RF and Microwave Power FETS (Cambridge University Press, 2007), and has authored or co-authored of over a dozen papers. 


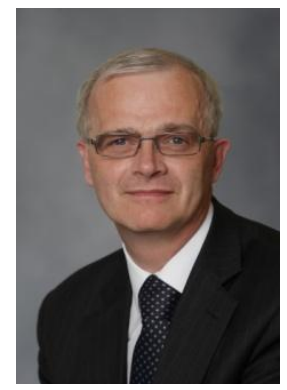

Christopher M. Snowden (S'82-M'82-SM'91F'96) received the B.Sc. (Hons.), M.Sc. and Ph.D. degrees from the University of Leeds, U.K. After graduating in 1977, he worked as an Applications Engineer for Mullard. His Ph.D. studies were later conducted in association with Racal-MESL .

$\mathrm{He}$ held the personal Chair of Microwave Engineering at the University of Leeds from 1992 to 2005. During the period 1995-1998, he was Head of the Department and subsequently Head of the School of Electronic and Electrical Engineering. He was the first Director of the Institute of Microwaves and Photonics located in the School. He was a Consultant to M/A-COM Inc., from 1989 to 1998. In 1998, he joined Filtronic plc as Director of Technology and was promoted to Joint Chief Executive in 1999. He was Chief Executive of Filtronic ICS and Professor of Microwave Engineering at the University of Leeds until April 2005. He is now the ViceChancellor and Chief Executive of the University of Surrey. He is President of the Institution of Engineering and Technology and Vice-President of the Royal Academy of Engineering. His main research interests include semiconductor device modeling and microwave circuit technology and design. He has written eight books, over 300 refereed journal and conference papers, and many other articles.

Prof. Snowden was awarded the 1999 Microwave Prize and the 2009 Distinguished Educator Award of the IEEE Microwave Theory and Techniques Society. In 2004 he was awarded the Silver Medal of the Royal Academy of Engineering for his contributions to the compound semiconductor industry. He is a Fellow of the IEEE, Fellow of the Royal Society, a Fellow of the Royal Academy of Engineering, and a Fellow of the IET. He was a Distinguished Lecturer for the IEEE Electron Devices Society until 2005. 